\title{
Author Correction to: TRIM29 negatively controls antiviral immune response through targeting STING for degradation
}

Qijie Li', Liangbin Lin', Yanli Tong ${ }^{1}$, Yantong Liu², Jun Mou' ${ }^{1}$ Xiaodong Wang ${ }^{1}$, Xiuxuan Wang ${ }^{3}$, Yanqiu Gong ${ }^{3}$, Yi Zhao', Yi Liu', Bo Zhong ${ }^{4}$, Lunzhi Dai (1) ${ }^{3}$, Yu-Quan Wei ${ }^{2}$, Huiyuan Zhang ${ }^{1}$ and Hongbo Hu (D)

Correction to: Cell Discovery (2018) 4:13 https://doi. org/10.1038/s41421-018-0010-9, published online 20 March 2018

In the original publication of this article ${ }^{1}$ one of the author names was incorrect. In this correction article the correct and incorrect name are indicated.

Previous incorrect name:

Yu-Quan We

Corrected name:

Yu-Quan Wei

The original publication has been updated.

\section{Author details}

'Department of Rheumatology and Immunology, State Key Laboratory of Biotherapy and Collaborative Innovation Center for Biotherapy, West China
Hospital, Sichuan University, Chengdu 610041, China. ${ }^{2}$ Department of General Practice and Lab of PTM, State Key Laboratory of Biotherapy and Collaborative Innovation Center for Biotherapy, West China Hospital, Sichuan University, Chengdu 610041, China. ${ }^{3}$ Cancer Center, State Key Laboratory of Biotherapy and Collaborative Innovation Center for Biotherapy, West China Hospital, Sichuan University, Chengdu 610041, China. ${ }^{4}$ School of Life Science, Wuhan University, Wuhan, China

Received: 12 April 2018 Accepted: 12 April 2018

Published online: 31 May 2018

References

1. Qijie, L. i. et al. TRIM29 negatively controls antiviral immune response through targeting STING for degradation. Cell Discov. 4, 13, https://doi.org/10.1038/ s41421-018-0010-9 (2018).

Correspondence: Huiyuan Zhang (hyzhang@scu.edu.cn) or

Hongbo Hu (hongbohu@scu.edu.cn)

'Department of Rheumatology and Immunology, State Key Laboratory of Biotherapy and Collaborative Innovation Center for Biotherapy, West China Hospital, Sichuan University, Chengdu 610041, China

2Department of General Practice and Lab of PTM, State Key Laboratory of Biotherapy and Collaborative Innovation Center for Biotherapy, West China Hospital, Sichuan University, Chengdu 610041, China

Full list of author information is available at the end of the article

(c) The Author(s) 2018

(c) (i) Open Access This article is licensed under a Creative Commons Attribution 4.0 International License, which permits use, sharing, adaptation, distribution and reproduction in any medium or format, as long as you give appropriate credit to the original author(s) and the source, provide a link to the Creative Commons license, and indicate if changes were made. The images or other third party material in this article are included in the article's Creative Commons license, unless indicated otherwise in a credit line to the material. If material is not included in the article's Creative Commons license and your intended use is not permitted by statutory regulation or exceeds the permitted use, you will need to obtain permission directly from the copyright holder. To view a copy of this license, visit http://creativecommons.org/licenses/by/4.0/. 